\title{
Vida saludable perdida por discapacidad aguda, crónica y muerte prematura en hipertensión arterial
}

\section{Disability adjusted life years lost due to acute, chronic disability and premature death in arterial hypertension}

\author{
Enrique Villarreal-Ríos ${ }^{1 *}$, Griselda Montoya-Cruz ${ }^{1,2}$, Emma R. Vargas-Daza ${ }^{1}$, Liliana Galicia-Rodríguez ${ }^{1}$, \\ Verónica Escorcia-Reyes ${ }^{3}$ y Laura A. Cu-Flores ${ }^{4}$ \\ ${ }^{1}$ Unidad de Investigación Epidemiológica y en Servicios de Salud Querétaro, Instituto Mexicano del Seguro Social (IMSS), Querétaro; ${ }^{2}$ Servicio de \\ Medicina Familiar, Unidad de Medicina Familiar No 9, IMSS, Querétaro; ${ }^{3}$ Unidad de Medicina Familiar No 6, IMSS, San Juan del Río, Querétaro; \\ ${ }^{4}$ Unidad de Medicina Familiar No 16, IMSS, Querétaro. México
}

\section{Resumen}

Objetivo: Determinar la vida saludable perdida por hipertensión arterial sin diabetes mellitus. Método: La vida saludable perdida se determinó a partir de la discapacidad crónica (enfermedad renal crónica, cardiopatía y evento vascular cerebral), la discapacidad aguda (crisis hipertensiva y emergencia hipertensiva) y la muerte prematura. Se identificaron la edad del diagnóstico, la edad de la complicación, la prevalencia de la complicación, la duración del evento agudo, el número de eventos agudos, el tiempo vivido con hipertensión, la edad de la muerte y la esperanza de vida. En todos los casos se aplicó una tasa de descuento del 3\%. La estimación se realizó por 100,000. Resultados: Cuando se utilizó como referencia el total de mujeres, la vida saludable perdida en ellas es de 198,498.28. Empleando como referencia el total de hombres, el valor para ellos es de 204,232.13. Si el referente es el total de la población, para las mujeres la vida saludable perdida es de 102,028.11 y para los hombres es de 99,256.98. Conclusiones: La vida sWaludable perdida por hipertensión arterial sin diabetes es diferente en hombres y mujeres; no obstante, tiene muchas aristas que deben abordarse.

Palabras clave: Años de vida saludables perdidos. Discapacidad. Esperanza de vida. Hipertensión arterial. Mortalidad.

\begin{abstract}
Objective: To determine the disability adjusted life years in arterial hypertension without diabetes mellitus. Method: Disability adjusted life years was determined from chronic disability (chronic kidney disease, heart disease and cerebral vascular event), acute disability (hypertensive crisis and hypertensive emergency) and premature death. Age of diagnosis, age of the complication, prevalence of the complication, duration of the acute event, number of acute events, time lived with hypertension, age of death and life expectancy were identified. In all cases a 3\% discount rate was applied, the estimate was made per 100,000. Results: When the total of women was used as a reference, the disability adjusted life years in women is 198,498.28. In men, using the total number of men as a reference, the value is 204,232.13. If the referent is the total population, in women the disability adjusted life years is 102,028.11 and in men 99,256.98. Conclusions: The disability adjusted life years in arterial hypertension without diabetes is different for men and women; the topic has many edges that must be studied.
\end{abstract}

Key words: Disability adjusted life. Disability. Life expectancy. Arterial hypertension. Mortality.

\section{Correspondencia:}

*Enrique Villarreal-Ríos

E-mail: enriquevillarrealrios@gmail.com
Fecha de recepción: 08-04-2020

Fecha de aceptación: 10-07-2020 DOI: $10.24875 /$ ACM. 20000140
Disponible en internet: 24-11-2020 Arch Cardiol Mex. 2021;91(2):202-207 www.archivoscardiologia.com 1405-9940 / @ 2020 Instituto Nacional de Cardiología Ignacio Chávez. Publicado por Permanyer. Este es un artículo open access bajo la licencia CC BY-NC-ND (http://creativecommons.org/licenses/by-nc-nd/4.0/). 


\section{Introducción}

Las enfermedades crónicas, como la hipertensión arterial, constituyen en la actualidad uno de los mayores retos que enfrentan los sistemas de salud en todo el mundo ${ }^{1,2}$. En la hipertensión arterial están involucradas la prevalencia, las complicaciones agudas y las complicaciones crónicas, manifestadas como crisis hipertensiva, emergencia hipertensiva, insuficiencia cardiaca, cardiopatía isquémica, evento vascular cerebral, nefropatía y retinopatía hipertensiva, que se traducen en pérdida de vida saludable ${ }^{3-6}$. Aunado a las complicaciones, la edad temprana de presentación de la hipertensión arterial aumenta la probabilidad de muerte prematura, lo que incide en la vida saludable perdida ${ }^{7-9}$. Las complicaciones y la muerte prematura se traducen en años de vida saludable perdidos; la estimación de este indicador contempla la suma de los años de vida perdidos por muerte prematura y los años de vida perdidos ajustados por discapacidad aguda o crónica ${ }^{10,11}$.

El planteamiento original de la estimación de años de vida saludables perdidos se propuso para tres grandes grupos: enfermedades transmisibles, enfermedades no transmisibles, y accidentes y lesiones. La hipertensión se ubicó en el grupo de enfermedades no transmisibles, en el subgrupo de enfermedades cardiovasculares. Ante esta propuesta se abre la posibilidad de identificar la vida saludable perdida por hipertensión arterial ${ }^{12-14}$.

En torno a la hipertensión arterial se debe reconocer su alta incidencia en población de 20 años y más $(31.1 \%)^{6}$, y aunado a ello la modificación de la evolución cuando coexiste con otras patologías ${ }^{15}$.

En este contexto, el objetivo del artículo es determinar la vida saludable perdida por hipertensión arterial sin diabetes mellitus.

\section{Método}

Diseño transversal descriptivo en pacientes con hipertensión arterial sin diabetes mellitus. El tiempo de recolección de la información fue de mayo a junio de 2019, en las unidades médicas de un sistema de seguridad social de Querétaro, México. Se incluyeron pacientes con hipertensión arterial esencial diagnosticada por el médico familiar y en control en el primer nivel de atención.

El cálculo de la vida saludable perdida se realizó para la discapacidad aguda, la discapacidad crónica y la muerte prematura.

Para la estimación de la discapacidad aguda y crónica se analizaron los expedientes de 270 pacientes con hipertensión arterial sin diabetes. El tamaño de muestra se calculó con la fórmula de promedios para población finita, con un nivel de confianza del $95 \%(Z \alpha=1.64)$ para una zona de rechazo de la hipótesis nula, asumiendo como hipótesis que el promedio de años de vida saludable perdidos por individuo fue superior a 11 , con desviación estándar de 1.41 ( $\mathrm{s}=1.41$ ), margen de error de $0.14(\mathrm{~d}=0.14)$ y total de población de 26,000 $(\mathrm{N}=26,000)$. La técnica muestral fue por casos consecutivos, empleando como marco el listado de pacientes hipertensos adscritos a la unidad médica.

La información generada a partir del expediente clínico incluyó la edad actual del paciente, el sexo, la prevalencia de obesidad y la edad del paciente en el momento del diagnóstico de la hipertensión arterial.

Se incluyeron como parte de la discapacidad aguda la crisis hipertensiva y la emergencia hipertensiva; para la discapacidad crónica se consideraron la enfermedad renal crónica, la cardiopatía y el evento vascular cerebral.

Con la información registrada en el expediente clínico se identificaron la edad del paciente al momento del diagnóstico de la complicación, la prevalencia de la complicación, la duración en días del evento agudo y el número de eventos agudos al año.

El tiempo vivido (en años) con la complicación se estimó a partir de la edad de presentación de esta y la edad de la muerte.

Para estimar la edad de la muerte en la población con hipertensión arterial se asumió que en ella la expectativa de vida son 10 años menos que en la población general, adoptando como esperanza de vida de la población mexicana 77.8 años para las mujeres y 72.6 años para los hombres ${ }^{16}$.

Los años vividos con hipertensión arterial se obtuvieron de restar a la edad de la muerte la edad del diagnóstico de hipertensión arterial. Se asumió la esperanza de vida de la población japonesa: 82.5 años para las mujeres y 80.0 años para los hombres ${ }^{17}$.

Para identificar la vida saludable perdida por discapacidad aguda y crónica se convocó a un panel de cinco expertos con especialidad en medicina familiar (responsables del control mensual del paciente con hipertensión arterial), con más de 10 años de experiencia en la práctica clínica, para que identificaran el porcentaje de vida saludable perdido por tipo de discapacidad aguda y crónica, ponderado por la prevalencia del estadio de cada complicación. A la estimación de la vida saludable perdida por discapacidad aguda o crónica se le aplicó la tasa de descuento de $3 \%$. 
Tabla 1. Edad del diagnóstico, edad de la muerte y años vividos con hipertensión arterial por sexos

\begin{tabular}{|l|c|c|}
\hline Indicador & Mujer & Hombre \\
\hline Edad del diagnóstico (años) & 43.61 & 40.12 \\
\hline Edad de la muerte (años) & 67.80 & 62.60 \\
\hline Años vividos con hipertensión* & 24.19 & 22.48 \\
\hline
\end{tabular}

Supuesto: se asumió que la población con hipertensión arterial vive 10 años menos que la general. Esperanza de vida en población mexicana: 77.8 para las mujeres y 72.6 para los hombres ${ }^{16}$.

*Edad de la muerte menos edad del diagnóstico de hipertensión arterial.

Para la estimación de la vida saludable perdida por muerte prematura se adoptó la esperanza de vida al nacer para la población de Japón (82.5 años para las mujeres y 80.0 años para los hombres) ${ }^{17}$. Se identificó la edad de la muerte en la población (ya descrito) y se determinaron los años perdidos por muerte prematura. Se aplicó la tasa de descuento del $3 \%$.

Se proyectaron escenarios, empleando para ello la población de México, 119,530,733 habitantes (INEGI), el $51.4 \%$ mujeres $(61,474,620)$ y el $48.6 \%$ hombres $(58,056,133)$, el porcentaje de mujeres de 20 años 0 más, $33.5 \%(40,042,802)$, y el porcentaje de hombres de 20 años o más, $29.9 \%(35,739,695)^{13}$. También se adoptó la prevalencia $(31.5 \%)$ de hipertensión arterial en población de 20 años o más ${ }^{6}$.

Las fuentes de información fueron los pacientes, los registros institucionales y los expertos. En todos los casos se contó con el consentimiento informado de los entrevistados.

El proyecto fue registrado ante el Comité de Investigación Institucional y se realizó con recursos propios de la institución.

\section{Resultados}

El promedio de edad de la población encuestada fue de 45.77 años (intervalo de confianza del 95\% [IC95\%]: 42.86-48.68) en los hombres y de 50.64 años (IC95\%: 48.34-52.92) en las mujeres. La prevalencia del sexo femenino fue del 54.8\% (IC95\%: 48.8-60.7) y la del masculino fue del $45.2 \%$ (IC95\%: 39.3-51.2). La prevalencia de sobrepeso y obesidad en los hombres fue del $84.43 \%$ (IC95\%: 77,9-90.9) y en las mujeres fue del 89.1\% (IC5\%: 84.0-94.2).

El promedio de edad al momento del diagnóstico de hipertensión arterial fue de 43.61 años en las mujeres y de 40.12 años en los hombres. En la tabla 1 se indican la edad de la muerte y el tiempo vivido con hipertensión arterial, por sexos.

La edad de diagnóstico de cardiopatía fue de 46.50 años en las mujeres y 53.67 años en los hombres. La prevalencia de cardiopatía fue del $6.08 \%$ en las mujeres y del $7.38 \%$ en los hombres. El tiempo vivido con cardiopatía en mujeres fue de 14.13 años y en los hombres fue de 8.93 años. En la tabla 2 se presenta esta información para enfermedad renal crónica y evento vascular cerebral.

La edad de presentación de la primera crisis hipertensiva fue de 46.75 años en las mujeres y de 41.87 años en los hombres. En la tabla 2 se presentan el resto de las características de la discapacidad aguda.

La evaluación proporcionada por los expertos en torno a la vida saludable perdida por discapacidad crónica y aguda varió: $90.0 \%$ para la crisis hipertensiva y $1.97 \%$ para la enfermedad renal crónica. En la tabla 2 se presenta esta información para el resto de las complicaciones.

Los años perdidos por muerte prematura aplicando la tasa de descuento del 3\% son 9.52 en las mujeres y 10.40 en los hombres. En la tabla 3 se presentan los supuestos adoptados para estimar la vida perdida por muerte prematura.

El total de años perdidos por 100,000 habitantes es de 102,028.11 en las mujeres y 99,256.82 en los hombres. La muerte prematura es la que contribuye con más años de vida saludables perdidos, 100,453.60 en las mujeres y $97,985.83$ en los hombres. En la tabla 4 se muestran los años saludables perdidos por tipo de discapacidad aguda y crónica.

\section{Discusión}

El peso de la enfermedad ha sido ampliamente estudiando ${ }^{18,19}$, pero es una realidad que en la actualidad la multipatología es un escenario común; en particular, en los pacientes hipertensos existe una fuerte coexistencia con la diabetes, distinción que en el análisis de la vida saludable perdida pocas veces se aborda. En ello radica la importancia del estudio que aquí se presenta, en el que se muestra la vida saludable perdida por hipertensión arterial sin diabetes mellitus y en particular se identifica la contribución de la discapacidad aguda, la discapacidad crónica y la muerte prematura en la pérdida de vida saludable.

Se debe señalar que es una fortaleza del trabajo la estimación realizada por los expertos para determinar la vida saludable perdida, y dentro de ella la identificación de la prevalencia de los estadios de las complicaciones 
Tabla 2. Características de la discapacidad crónica y de la discapacidad aguda por sexos y según la evaluación de los expertos de pérdida de vida saludable

\begin{tabular}{|c|c|c|c|c|c|c|}
\hline \multirow[t]{3}{*}{ Característica } & \multicolumn{6}{|c|}{ Discapacidad crónica } \\
\hline & Mujer & Hombre & Mujer & Hombre & Mujer & Hombre \\
\hline & \multicolumn{2}{|c|}{ Enfermedad renal crónica } & \multicolumn{2}{|c|}{ Cardiopatía } & \multicolumn{2}{|c|}{$\begin{array}{c}\text { Evento vascular } \\
\text { cerebral }\end{array}$} \\
\hline Edad de presentación (años) & 57.01 & 50.91 & 46.50 & 53.67 & 47.00 & 56.00 \\
\hline Años vividos con la complicación & 10.73 & 11.69 & 14.13 & 8.93 & 10.73 & 11.69 \\
\hline Prevalencia de la complicación (\%) & 10.14 & 18.85 & 6.08 & 7.38 & 2.70 & 1.64 \\
\hline Vida saludable perdida (\%) & \multicolumn{2}{|c|}{1.97} & \multicolumn{2}{|c|}{10.80} & \multicolumn{2}{|c|}{25.60} \\
\hline \multirow[t]{3}{*}{ Característica } & \multicolumn{6}{|c|}{ Discapacidad aguda } \\
\hline & Mujer & Hombre & Mujer & \multicolumn{3}{|c|}{ Hombre } \\
\hline & \multicolumn{2}{|c|}{ Crisis hipertensiva } & \multicolumn{4}{|c|}{ Emergencia hipertensiva } \\
\hline Edad de primera presentación (años) & 46.75 & 41.87 & 55.12 & & 52.00 & \\
\hline Años vividos con la complicación & 21.05 & 20.73 & 12.68 & & 10.60 & \\
\hline Prevalencia de la complicación (\%) & 3.38 & 6.56 & 1.35 & & 1.64 & \\
\hline Duración el evento agudo (días) & 1.00 & 1.00 & 1.00 & & 1.00 & \\
\hline Número de eventos al año & 1.00 & 1.00 & 1.00 & & 1.00 & \\
\hline Vida saludable perdida (\%) & 90.0 & & 90.0 & & & \\
\hline
\end{tabular}

Tabla 3. Supuestos adoptados para estimar la vida saludable perdida por muerte prematura

\begin{tabular}{|l|c|c|}
\hline Característica & Mujer & Hombre \\
\hline Esperanza de vida (años)* & 82.50 & 80.00 \\
\hline Edad de la muerte (años) & 67.80 & 62.60 \\
\hline Años perdidos por muerte prematura & 14.70 & 17.40 \\
\hline Años perdidos por muerte prematura ajustado ${ }^{\dagger}$ & 9.52 & 10.40 \\
\hline
\end{tabular}

*Se adoptó como referencia la esperanza de vida de la población de Japón para mujeres y hombres ${ }^{17}$.

TTasa de descuento del $3 \%$.

crónicas y la asignación diferenciada de pérdida de vida saludable por estadio, información que permite tener un valor ponderado. Es verdad que existe la propuesta de The Global Burden of Disease and Injury para medir la discapacidad, pero la realidad es que los métodos son muy variados, aunque en todos ellos el principio es el mismo: identificar la gravedad de la discapacidad, propuesta que se asumió en este trabajo 20,21 .

En los pacientes con hipertensión arterial sin diabetes mellitus, la dimensión que más contribuye a la vida saludable perdida es la muerte prematura, tendencia que coincide con lo descrito en la literatura ${ }^{18}$. La diferencia en la contribución de la discapacidad aguda y de la discapacidad crónica es muy evidente; sin embargo, hay que ser consciente de que llegar a la muerte prematura es una consecuencia de la discapacidad aguda o crónica, y por lo tanto el manejo de la discapacidad aguda o crónica es un área de oportunidad para los servicios de salud en aras de disminuir el peso de la enfermedad en la hipertensión arterial.

En realidad, la vida saludable perdida por hipertensión arterial a quien evalúa es a la población, pero indirectamente es un reflejo del actuar de los servicios de salud, ya que estos se encuentran involucrados en el cuidado de la salud de la población.

La estimación de la vida saludable perdida por hipertensión arterial sin diabetes define la discapacidad como aquellos eventos considerados complicaciones agudas o crónicas de la hipertensión, y estos son los que aportan vida saludable perdida al indicador. En este momento, el cuestionamiento lógico se establece en torno a lo que se mide; se está midiendo la complicación o se está midiendo la hipertensión. En realidad, cualquiera de las complicaciones es una afección 
Tabla 4. Años de vida saludable perdidos por discapacidad crónica, discapacidad aguda, muerte prematura y total en mujeres y hombres en tres escenarios

\begin{tabular}{|c|c|c|c|c|c|c|}
\hline \multirow[t]{3}{*}{ Indicador* } & \multicolumn{2}{|c|}{ Escenario 1} & \multicolumn{2}{|c|}{ Escenario 2} & \multirow{2}{*}{\multicolumn{2}{|c|}{$\begin{array}{c}\text { Escenario } 3 \\
\text { Toda la población }\end{array}$}} \\
\hline & \multirow{2}{*}{$\begin{array}{c}\text { Mujeres de } 20 \\
\text { años y más }\end{array}$} & \multirow{2}{*}{$\begin{array}{l}\text { Hombres de } 20 \\
\text { años y más }\end{array}$} & \multirow{2}{*}{$\begin{array}{c}\text { Todas las } \\
\text { mujeres }\end{array}$} & \multirow{2}{*}{$\begin{array}{l}\text { Todos los } \\
\text { hombres }\end{array}$} & & \\
\hline & & & & & Mujeres & Hombres \\
\hline $\begin{array}{l}\text { Discapacidad crónica } \\
\text { Nefropatía } \\
\text { Cardiopatía } \\
\text { Secuela evento vascular cerebral } \\
\text { Total }\end{array}$ & $\begin{array}{c}491.54 \\
2,347.80 \\
1,703.32 \\
4,542.66\end{array}$ & $\begin{array}{c}967.87 \\
1,721.68 \\
1,093.69 \\
3,783.24\end{array}$ & $\begin{array}{c}320.36 \\
1,530.18 \\
1,110.14 \\
2,960.68\end{array}$ & $\begin{array}{c}595.46 \\
1,059.22 \\
672.87 \\
2,327.55\end{array}$ & $\begin{array}{c}164.66 \\
786.51 \\
570.61 \\
1,521.79\end{array}$ & $\begin{array}{c}289.39 \\
514.78 \\
327.01 \\
1,131.19\end{array}$ \\
\hline $\begin{array}{l}\text { Discapacidad aguda } \\
\text { Crisis hipertensiva } \\
\text { Emergencia hipertensiva } \\
\text { Total }\end{array}$ & $\begin{array}{c}148.24 \\
9.15 \\
157.39\end{array}$ & $\begin{array}{c}457.68 \\
9.87 \\
467.55\end{array}$ & $\begin{array}{c}96.62 \\
5.96 \\
102.58\end{array}$ & $\begin{array}{c}281.58 \\
6.07 \\
287.65\end{array}$ & $\begin{array}{c}49.66 \\
3.06 \\
52.73\end{array}$ & $\begin{array}{c}136.85 \\
2.95 \\
139.80\end{array}$ \\
\hline $\begin{array}{l}\text { Muerte prematura } \\
\text { Total }\end{array}$ & $\begin{array}{l}299,861.48 \\
299,861.48\end{array}$ & $\begin{array}{l}327,711.81 \\
327,711.81\end{array}$ & $\begin{array}{l}195,435.01 \\
195,435.01\end{array}$ & $\begin{array}{l}201,616.94 \\
201,616.94\end{array}$ & $\begin{array}{l}100,453.60 \\
100,453.60\end{array}$ & $\begin{array}{l}97,985.83 \\
97,985.83\end{array}$ \\
\hline $\begin{array}{l}\text { Total } \\
\text { Total }\end{array}$ & $304,561.54$ & $331,962.60$ & $198,498.28$ & $204,232.13$ & $102,028.11$ & $99,256.82$ \\
\hline
\end{tabular}

*Por 100,000 .

independiente que podría tener otro origen, pero en el caso de la hipertensión arterial se presentan como un evento secundario al proceso hipertensivo, y en consecuencia la vida saludable perdida se le debe atribuir. Lo mismo sucede con la mortalidad prematura: la muerte se presenta por la enfermedad renal crónica o por la cardiopatía, pero estas son secundarias a la hipertensión arterial.

Los reportes de vida saludable perdida por cardiopatía identifican 841,187 años saludables perdidos en los hombres y 537,422 en las mujeres ${ }^{18}$. Será necesario dilucidar si la vida saludable perdida por hipertensión arterial forma parte de esa contabilización, y el mismo planteamiento aplica para la vida saludable perdida por enfermedad renal crónica y evento vascular cerebral, entre otros.

Cuando se compara la vida saludable perdida entre hombres y mujeres se aprecia una mayor carga en los hombres cuando se utiliza como referente el grupo de hombres o el de mujeres. Esto coincide con los reportes de la literatura ${ }^{18}$. Sin embargo, cuando se utiliza como referencia la población total, la tendencia se modifica.

Es verdad que en este trabajo la estimación de la vida saludable perdida por discapacidad crónica no incluye la isquemia cardiaca ni la retinopatía hipertensiva, pero no por ello se descalifican los resultados; será necesario complementarlos en otras investigaciones.

Indudablemente, la estimación de la vida saludable perdida se modificará en función de la población empleada como referencia, escenario evidente cuando se presentan los resultados para toda la población, para mujeres y hombres, y para mayores de 20 años. Es verdad que ya está establecido el referente para realizar la comparación con otras poblaciones, pero el análisis de los escenarios es obligado como parte del estudio del tema.

Finalmente, la esencia de la investigación es la generación del conocimiento por el conocimiento, pero al final del camino el conocimiento es empleado en beneficio de la sociedad, y en ese tenor se debe tener claro en qué o para qué se puede utilizar el conocimiento de la vida saludable perdida por hipertensión arterial sin diabetes mellitus, y aunado a ello, quién es el usuario del conocimiento: el clínico, el epidemiólogo o el directivo tomador de decisiones para normar políticas públicas; la respuesta parece rebasar la pretensión de este artículo.

\section{Conclusiones}

La vida saludable perdida por hipertensión arterial sin diabetes es diferente en hombres y mujeres; no obstante, el tema tiene muchas aristas que deben abordarse.

\section{Conflictos de intereses}

Ninguno. 


\section{Financiamiento}

La presente investigación no ha recibido ninguna beca específica de agencias de los sectores público, comercial o sin ánimo de lucro.

\section{Responsabilidades éticas}

Protección de personas y animales. Los autores declaran que para esta investigación no se han realizado experimentos en seres humanos ni en animales.

Confidencialidad de los datos. Los autores declaran que han seguido los protocolos de su centro de trabajo sobre la publicación de datos de pacientes.

Derecho a la privacidad y consentimiento informado. Los autores declaran que en este artículo no aparecen datos de pacientes.

\section{Bibliografía}

1. Instituto Mexicano del Seguro Social. Diagnóstico y tratamiento de la hipertensión arterial en el adulto mayor. Guía de evidencias y recomendaciones. Guía de práctica clínica. México: Instituto Mexicano del Seguro Social; 2017. Disponible en: http://www.imss.gob.mx/sites/all/statics/ guiasclinicas/238GER.pdf.

2. Secretaría de Integración y Desarrollo del Sector Salud. Informe sobre la salud de los mexicanos 2015. Diagnóstico general de la salud poblacional. México: Secretaría de Salud; 2015. Disponible en: https://www. gob.mx/cms/uploads/attachment/file/64176/INFORME_LA_SALUD_DE LOS_MEXICANOS_2015_S.pdf.

3. Soto Estrada G, Moreno Altamirano L, Pahua Díaz D. Panorama epidemiológico de México, principales causas de morbilidad y mortalidad. Revista de la Facultad de Medicina de la UNAM. 2016;59:8-22.

4. Secretaría de Salud, Instituto Nacional de Salud Pública. Encuesta Nacional de Salud y Nutrición de Medio Camino 2016. Informe final de resultados. México: Secretaría de Salud; 2016. Disponible en: https:// www.gob.mx/cms/uploads/attachment/file/209093/ENSANUT.pdf.

5. Rivero O, Martínez A. Hipertensión arterial. Prevalencia y factores de riesgo en la población de un consultorio de Morón. Convenio Internacional de Salud. Cuba; 2018. Disponible en: http://www.convencionsalud2018.sld.cu/index.php/connvencionsalud/2018/paper/view/364/430
6. Campos Nonato I, Hernández Barrera L, Pedroza Tobías A, Medina C, Barquera S. Hipertensión arterial en adultos mexicanos: prevalencia, diagnóstico y tipo de tratamiento. Ensanut MC 2016. Salud Pública de México. 2018;60:233-43.

7. Ramos MV. Hipertensión arterial: novedades de las guías 2018. Rev Urug Cardiol. 2019;34:53-60.

8. Santeiro Pérez L, Valdés Gómez M, Díaz Quiñones J, Díaz Marrero J, Sarmiento Albelo $Y$. Años de vida potencialmente perdidos por primeras causas de muerte. Rev Finlay. 2018;8(3). Disponible en: http://revfinlay. sld.cu/index.php/finlay/article/view/602.

9. Pompa SG, Duany LF, Tamayo BDC, Álvarez A, Fonseca RL. Nefropatía hipertensiva: factores de riesgo en pacientes pertenecientes al policlínico Jimmy Hirzel. Revista 16 de Abril. 2018;57:80-8. Disponible en: https:// www.medigraphic.com/pdfs/abril/abr-2018/abr18268d.pdf.

10. Frenk J, Ruelas E, Bobadilla JL, Zurita B, Lozano R, González Block MG, et al. Economía y salud: propuestas para el avance del sistema de salud en México. Informe final. México D.F.: Fundación Mexicana para la Salud; 1994.

11. Lozano R, Franco Marina F, Solís P. El peso de la enfermedad crónica en México. Salud Publica de México. 2007;49:E283-7.

12. Serra Valdés M, Serra Ruiz M, Viera García M. Las enfermedades crónicas no transmisibles: magnitud actual y tendencias futuras. Rev Finlay. 2018;8(2). Disponible en: http://scielo.sld.cu/scielo.php?script=sci_arttext\&pid=S2221-24342018000200008\&lng=es.

13. Instituto Nacional de Estadística y Geografía. Mujeres y hombres en México 2018. México: Instituto Nacional de Estadística y Geografía; 2018. Disponible en: http://cedoc.inmujeres.gob.mx/documentos_download/MHM_2018.pdf.

14. Ríos Blancas MJ, Cahuana Hurtado L, Lamadrid Figueroa H, Lozano R. Cobertura efectiva del tratamiento de la hipertensión arterial en adultos en México por Entidad Federativa. Salud Pública de México. 2017;59: 154-64.

15. Araya-Orozco M. Hipertensión arterial y diabetes mellitus. Rev Costarric Cienc Med. 2004;25:65-71.

16. Instituto Nacional de Geografía y Estadística. Esperanza de vida. México. Disponible en: http://cuentame.inegi.org.mx/poblacion/esperanza.aspx?tema=P.

17. Murruy C. Cuantificación de la carga de enfermedad: la base ética del cálculo de los años de vida ajustados en función de la discapacidad. Bol Oficina Sanit Panam. 1995;118:221-43.

18. Lozano R, Gómez Dantés H, Garrido Latorre F, Jiménez Corona A, Campuzano Rincón JC, Franco Marina F, et al. La carga de enfermedad, lesiones, factores de riesgo y desafíos para el sistema de salud en México. Salud Pública de México. 2013:55:580-94.

19. Murray C, López A. Quantifying disability: data, methods and results. WHO Bulletin OMS. 1994;72:481-94.

20. Organización Mundial de la Salud, Banco Mundial. Informe mundial sobre la discapacidad. Malta: Organización Mundial de la Salud; 2011. Disponible en: https://www1.paho.org/arg/images/Gallery/Informe_spa.pdf.

21. Murray C, López A. The global burden of disease and injury series. Cambridge: World Health Organization; 1996. Disponible en: https://apps. who.int/iris/bitstream/handle/10665/41864/0965546608_eng.pdf?sequence $=1$ \&isAllowed $=y$. 